\title{
Level of Adherence to Feeding Guidelines for Infants Born to Mothers Living with HIV in Rakai District, Uganda
}

\author{
Nwanna Uchechukwu Kevin *
}

Department of Health Sciences, Victoria University, Uganda

\begin{abstract}
Introduction: There are a number of challenges to both the mother and the healthcare workers due to the risk of Human Immunodeficiency Virus (HIV) transmission as a result of infant feeding which poses a risk of HIV transmission. Proper and sufficient nutrition is crucial for child growth and development, and throughout the lifespan of the child. The focus of the Uganda national health sector is to promote health, disease prevention, early diagnosis and treatment of disease. National minimum health care package consists of Maternal and Child Health; Prevention, management, and control of communicable diseases; Prevention, management, and control of noncommunicable diseases; Health promotion and community health initiatives.

Objective: The purpose of this study was to determine the level of adherence to feeding guidelines for infants born to mothers living with HIV in Rakai District, Uganda.

Methodology: A descriptive cross-sectional study design was used with a sample size of 138 respondents and employing a quantitative method of inquiry. A non-probability sampling strategy called consecutive sampling was used to sample the mothers with HIV. Data were analyzed using SPSS to generate descriptive analysis data.

Results: Out of 135 HIV mothers interviewed, 69 (51.1\%) adhered to breastfeeding guidelines for infants born to HIV mothers.

Conclusion: 5 in every 10 HIV mothers adhere to breastfeeding guidelines for infants born to HIV mothers.

Recommendations: Timely re-orientation of the mothers and family about the infant feeding, proper monitoring by the health workers.
\end{abstract}

Keywords: Infants; Feeding; HIV; Mothers; Uganda

\section{INTRODUCTION}

Uganda policy and guidelines on infant and young child feeding include: Parents shall be counseled and supported to introduce adequate, safe and appropriate complementary feeding when the child is six months old. However, parents can breastfeed their infants for more than two (2) years. All HIV negative mothers and those of unknown HIV status shall be counseled and supported to exclusively breastfeed their infants for the first six months of the child's life. Pregnant women and lactating mothers shall be appropriately cared for and encouraged to consume nutritious foods. It's highly recommended that HIV positive mothers exclusively breastfeed their children in the first six (6) months unless they can afford and sustain replacement feeding when recommended. Children born to mothers living with HIV shall be tested for HIV infection at 10 weeks of age [1].

In an area where under-fives suffer from diarrhea, malnutrition, and pneumonia, breastfeeding contributes to the foundation of child health. In addition, due to the high mortality of underfives mothers are encouraged to breastfeed exclusively during the first six (6) months and gradually introduce complementary foods for more than two years [2]. In 2010, WHO for the first time recommended ARV drug interventions to prevent postnatal transmission of HIV through breastfeeding [3]. In 2010, WHO adopted a public health approach, recommending that national

Corresponding author: Nwanna Uchechukwu Kevin, Department of Health Sciences, Victoria University, Uganda, Tel: +256778092462; E-mail: uchenwanna1@gmail.com

Received date: June 08, 2019; Accepted date: July 24, 2019; Published date: August 03, 2019

Citation: Kevin NU (2019) Level of Adherence to Feeding Guidelines for Infants Born to Mothers Living with HIV in Rakai District, Uganda. J Trop Dis 7:327.

Copyright: (c) 2019 Kevin NU. This is an open-access article distributed under the terms of the Creative Commons Attribution License, which permits unrestricted use, distribution and reproduction in any medium, provided the original author and source are credited. 
authorities promote and support one feeding practice for all women living with HIV accessing care in public health facilities. This was a major paradigm shift.

As part of the EMTCT service program, mothers living with HIV in Rakai district are given infant feeding counseling for the prevention of HIV transmission to infants through breast milk by the health workers at the pre-natal visits and are then sometimes provided with follow-up counseling during the subsequent visits [4]. However, the rate of adherence to breastfeeding guidelines (exclusive breastfeeding, complementary feeding, and termination of breastfeeding) particularly among HIV positive mothers has been reported to be below 30\% [5]. This could lead to the high vertical transmission rates and without appropriate interventions meant to increase adherence to the WHO exposed infant feeding guidelines among HIV positive mothers, cases of vertical transmission during the first six months of infants are bound to increase hence increased infant mortality rates due to HIV.

2010, global WHO HIV and infant feeding guidelines were updated to recommend that, in settings in which diarrhea, pneumonia and under nutrition were still common causes of infant and child mortality, national health authorities should, while providing ARV drugs, promote and support breastfeeding among women and mothers living with HIV [3]. HIV positive mothers are highly encouraged to breastfeed exclusively their infants during the first six (6) months and to keep up with it for one year. Mothers living with HIV should then consider stopping breastfeeding at 12 months if they are able to provide a nutritionally adequate and safe diet without breast milk. The guidelines noted that, for women living in food-insecure regions, continuing breastfeeding beyond 12 months may still be important for the child to achieve an adequate diet. The recommendation to breastfeed until 12 months of age and then consider whether to either stop breastfeeding or continue breastfeeding for longer was based on four considerations.

Adherence to infant-feeding recommendations is critical for the Human Immunodeficiency Virus (HIV)-free survival of infants of HIV-infected women. HIV-infected mothers in developing countries often face complex challenges which influence their choice of infant-feeding practice [6]. Mothers who are unable to cope with the challenges may succumb to practices which fall outside the infant feeding recommendations, thus jeopardizing their infants "HIV-free survival".

Based on accumulating evidence on the adverse effects of the avoidance or early cessation of breastfeeding in developing countries, and the benefits of antiretroviral (ART) interventions in reducing the Mother-To-Child Transmission (MTCT) of HIV through breast milk, the World Health Organization (WHO) [7], released the "rapid advice" guideline, i.e. revised WHO principles and recommendations on infant feeding in the context of HIV in 2009. The use of prophylaxis during the breastfeeding period until one week after exposure to breast milk has stopped is recommended in this guideline. It also recommends that HIV-infected mothers intending to breastfeed should do so exclusively for the first six months, and should introduce appropriate complementary food thereafter, and continue breastfeeding until the infant is 12 months of age.
A study to investigate the infant feeding practices amongst HIVpositive and -negative mothers (0-9 months postpartum) and describe the association between infant feeding practices and HIV- free survival was carried out. This study revealed that despite adequate feeding practices was low among mothers who were both negative and positive, mothers who were positive to HIV were more likely to accept safer feeding practices for their infants and this was as a result of the counseling given to them by the healthcare workers during the EMTCT program [8].

\section{METHODOLOGY}

\section{Study design}

This study design was a descriptive cross-sectional and analytical study with quantitative data collection methods. The crosssectional study enabled the researcher to collect all the required data at a point; the research objective was used to design questions that necessitated the collection and analysis of the data [9].

\section{Study population}

The study population was among mothers living with HIV in Rakai district. It was estimated that 1 in every 10 mothers in Rakai district is living with HIV [10]. In Rakia district 23 of the health centers and two hospitals were offering services for Prevention of Mother To Child Transmission (PMTCT) [11]. The data were collected at the 23 health center and the two hospitals consecutively.

\section{Inclusion criteria}

All mothers living with HIV in Rakai district and are willing to participate in the study.

\section{Exclusion criteria}

All HIV mothers in Rakai district who were unable to answer the question due to one condition or the other.

\section{Sample size calculation}

In a study, an estimate of the number of women living with HIV in Rakia district was available, which was around $10 \%$ [10]. The researcher determined the sample size using Kishi and Leslie formula, this method is used because the actual population of a woman living with HIV in Rakia district is unavailable. This method involves the equation below:

$\mathrm{N}=\frac{\mathrm{z}^{2} \mathrm{p.q}}{\mathrm{d}^{2}}$

Where

- $\mathrm{n}=$ desired sample size

- $\mathrm{Z}=$ standard normal deviate at a confidence level of $95 \%$ or 1.96

- $\mathrm{P}=$ proportion in the population with (10\%)

- $Q=1-p$ ( $P$ is the proportion of the population)

- $\mathrm{D}=$ degree of accuracy which is at (0.05) 
$\mathrm{N}=\frac{\mathrm{z}^{2} \cdot \mathrm{p} . \mathrm{q}}{\mathrm{d}^{2}} \quad \mathrm{~N}=\frac{1.96^{2} \times 0.1 \times(1-0.1)}{\mathrm{d}^{2}(0.05)^{2}}$

The $\mathrm{n}$ value=138; thus, the sample size for this study is 138

\section{Sampling procedures}

A non-probability sampling technique called consecutive sampling technique was used to assess the woman living with HIV in Rakia district; consecutive sampling technique involves selecting all individuals who agree to participate, provided they meet pre-established criteria until the number of desired subjects is achieved. This enabled the researcher to be more assessable to the respondents and achieve the desired sample size easily. This was done at the HIV clinic and other outreaches in the district.

\section{MEASUREMENT OF VARIABLES}

\section{Independent variable}

Mothers living with HIV: Attributes such as HIV status were controlled by using essential questions related to each attribute.

\section{Dependent variable}

Level of adherence to feeding guidelines: This was determined by asking whether the mothers exclusively breastfed for first six months of life, introducing appropriate complementary foods thereafter, and continue breastfeeding while being fully supported for ART adherence. Those mothers who adhere to the breastfeeding guidelines stated above were grouped under "adhere" and those who did not adhere were grouped under "not-adhere".

\section{DATA COLLECTION METHODS}

\section{Quantitative methods}

Data were collected using structured questionnaire and with the help of the research assistants. A semi-structured questionnaire was designed that contained all the questions related to the study objectives. The questionnaires were administered with the help of the research assistants to aid proper dissemination them.

\section{DATA MANAGEMENT}

\section{Quantitative analysis}

Data collected were analyzed using the Statistical Package for Social Sciences (version 22.0) [13]. Statistical calculations were done using descriptive analysis only. Results were presented in charts.

\section{Quality control (reliability and validity)}

The following quality control measures were employed in this study:

- To ensure validity, the researcher pre-tested the questionnaires prior to data collection

- During data collection, trained and experienced research assistants were employed to avoid interview bias

\section{Ethical considerations}

In order to uphold high ethical standards, the following measures were adhered to:

- Informed Consent-all respondents and participants were notified beforehand of the nature of the research, and their consent was sought prior to the completion of questionnaires

- Right to Privacy and Confidentiality-the identities of participants and their opinions were treated in the strictest of confidence [14]. A locked box was provided at each health facility for dropping off the completed questionnaires and this was emptied every third day, to prevent tracing back of the respondents

- Self-determination-all participants were notified beforehand about their right to ask questions, to refuse to give information, withdraw from the study at any given time and the freedom from any type of coercion

- A statement regarding the purpose of the inquiry was provided to all participants of the study, which outlined the participant's role in the study and how the information they provided was to be used

\section{Limitation of the study}

- The study may be limited by the information supplied, information bias does occur when the respondents prefer not to supply correct information example when administering the questionnaires to the respondents; this was curbed by emphasizing on the confidentiality of the information verbally and inclusion in the questionnaire

\section{RESULTS}

A total of 135 mothers living with HIV were sampled out of the 138 anticipated sample size indicating a response rate of $98 \%$.

\section{Adherence to breastfeeding guidelines for infants born to mothers living with HIV in Rakai district}

From Figure 1 below, out of 135 HIV mothers interviewed 69 $(51.1 \%)$ adhered to breastfeeding guidelines for infants born to HIV mothers while 66 (48.9\%) did not adhere.

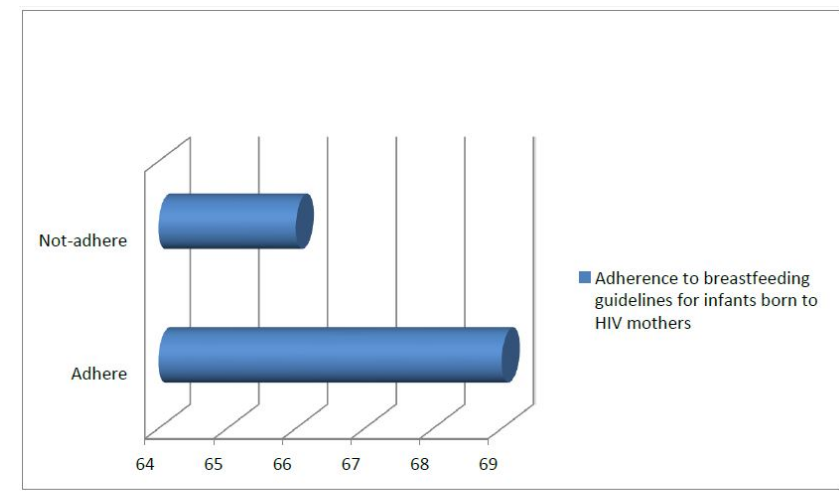

\section{Adherence to breastfeeding guidelines for} Infants born to HIV mothers

Figure 1: Bar chart displaying the population of adherence to breastfeeding guidelines for infants born to HIV mothers in Rakai district. 


\section{Breastfed the child with breast milk only for 6 months}

From Figure 2 below, out of 135 HIV mothers interviewed, 103 (76.3) of the mothers Breastfed the child with breast milk only for 6 months and 32 (23.7\%) did not breastfeed the child with breast milk only for 6 months.

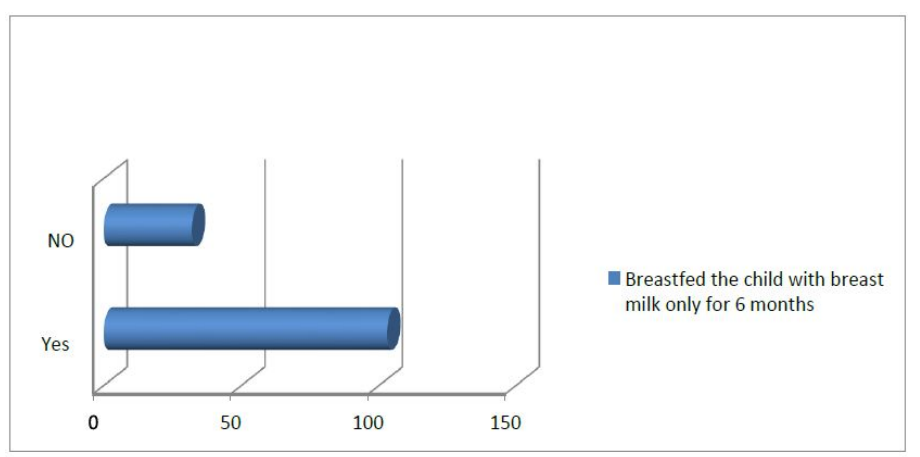

Breastfed the child with breast milk only for 6 months

Figure 2: Bar chart displaying the population mothers that Breastfed the child with breast milk only for 6 months in Rakai district.

\section{Appropriate complementary feed after 6 months}

As shown in Figure 3, the entire mothers interviewed 135 (100\%) introduced appropriate complementary feed after 6 months of breast milk only.

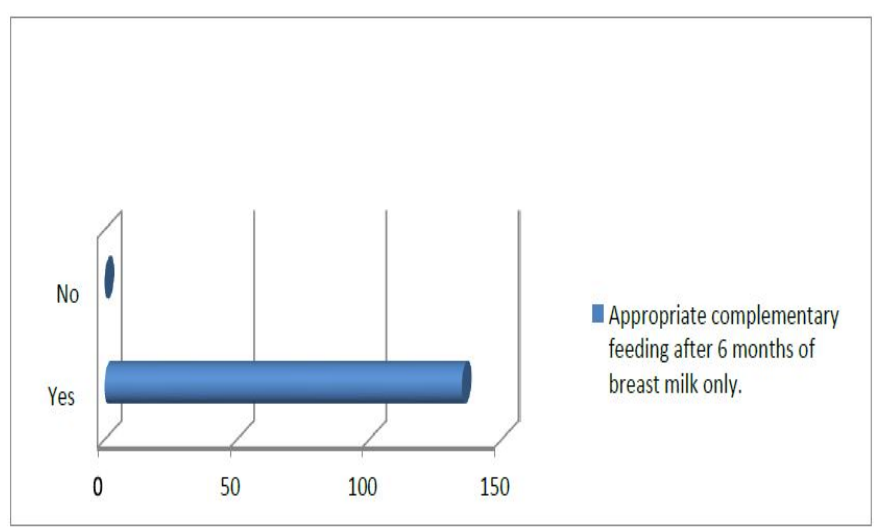

\section{Appropriate complementary feed after 6} Months of breast milk only.

Figure 3: Displaying the population of HIV mothers that introduce appropriate complementary feeding after 6 months of breast milk feeding only for their children.

\section{Duration of breastfeeding the infant}

Figure 4 shows that mothers who used up to 24 months to breastfeed were the majority of the respondents while those who used less than 6 months to breastfeed constituted the least respondents.

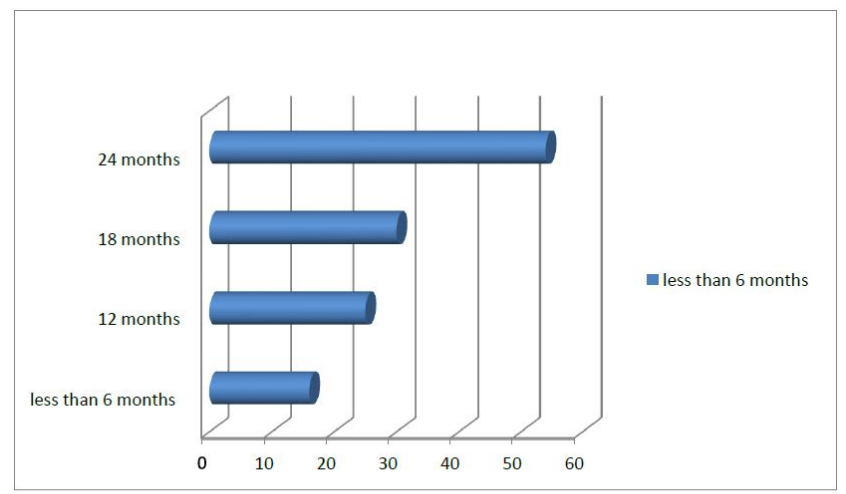

Duration of breastfeeding the infant

Figure 4: Bar chart displaying the various durations of breastfeeding of children born to mothers living with HIV in Rakai district.

\section{DISCUSSION}

\section{Adherence to breastfeeding guidelines for infants born to mothers living with HIV in Rakai district}

This research study found revealed that out of 135 HIV positive mothers interviewed $51.1 \%$ of them adhered to breastfeeding guidelines for infants born to HIV mothers while $48.9 \%$ of them did not adhere to the guidelines; this indicates that 5 in every $10 \mathrm{HIV}$ positive mothers adhered to breastfeeding guidelines for infants born to HIV mothers. In addition, this study reveals that the adherence to breastfeeding guidelines is on an average scale among the mothers living with HIV. Adherence to breastfeeding guidelines will help in building the immunity of the child, which will help in reducing the transmission of HIV from mothers to the child. The guideline was specifically designed in order to improve the health of the mother and child. The findings from this study is in line with a longitudinal descriptive study used to examine the adherence to infantfeeding choices made by Human Immunodeficiency Virus (HIV)-infected mothers at a Nigerian tertiary hospital, the results of the study revealed that $73 \%$ of the mothers adhered to breastfeeding guidelines for HIV mothers and 28\% did not adhere to breastfeeding guidelines for HIV mothers. Major reasons for non-adherence to their initial choice were fear of the Mother-To-Child Transmission (MTCT) of HIV (65\%) and nonaffordability of the formula (97\%) in the EBF and EFF groups, respectively [15].

In addition, a study was carried out to examine the feeding practices for infants belonging to HIV negative and positive mothers (0-9 months postpartum) and determine the relationship between the feeding practices of infant and the free survival of HIV. The results revealed that more HIV-positive mothers practiced exclusive breastfeeding as compared with HIV-negative mothers [16]. This shows that there is more to do in ensuring the acceptance of the infant feeding guidelines by the mothers because it has a very significant benefit in ensuring the rate of HIV transmission from mother to a child is greatly reduced. It also helps in the reduction of HIV burden globally. 


\section{CONCLUSION}

This study concluded that $51.1 \%$ of the HIV mothers adhered to breastfeeding guidelines for infants born to HIV positive mothers while $48.9 \%$ of the HIV positive mothers did not adhere to breastfeeding guidelines for infants born to HIV mothers, which indicates that 5 in every $10 \mathrm{HIV}$ positive mothers adhere to breastfeeding guidelines for infants born to HIV mothers.

\section{RECOMMENDATION}

Effective and efficient timely re-orientation of the mothers and family about the infant feeding approved guidelines will help mothers and family to have a better understanding of the breastfeeding guideline benefits; also regular campaigns and awareness will serve as a reminder to them always.

\section{REFERENCES}

1. Uganda IYCF Policy. Policy guidelines on infant and young child feeding-Ministry of Health. 2007.

2. World Health Organization. HIV/AIDS. 2016.

3. World Health Organization. Guidelines on HIV and infant feeding. 2010.

4. Kagaayi J, Gray RH, Brahmbhatt H, Kigozi G, Nalugoda F, Wabwire-Mangen F, et al. Survival of infants born to HIV-positive mothers, by feeding modality, in Rakai, Uganda. PLoS ONE. 2008;3:e3877.

5. Ministry of Health. Uganda demographic health survey 2011. 2012.

6. Leshbari SC, Blystad A, Moland KM. Difficult choices: Infant feeding experiences of HIV-positive mothers in Northern Tanzania. Sahara J. 2007;4:544-555.

7. World Health Organization. PMTCT strategic vision 2010-2015: preventing mother-to-child transmission of HIV to reach the UNGASS and Millennium Development Goals: Moving towards the elimination of paediatric HIV, December 2009. 2010.

8. Dinh TH, Delaney KP, Goga A, Jackson D, Lombard C, Woldesenbet $\mathrm{S}$, et al. Impact of maternal HIV seroconversion during pregnancy on early mother to child transmission of HIV (MTCT) measured at 4-8 weeks postpartum in South Africa 2011-2012: A national population-based evaluation. PloS One. 2015;10:e0125525.

9. Setia MS. Methodology series module 3: Cross-sectional studies. Ind J Dermatol. 2016;61:261.

10. Rakai district statistical. Abstract. 2009.

11. Kunihira NR, Nuwaha F, Mayanja R, Peterson S. Barriers to use of antiretroviral drugs in Rakai district of Uganda. Afr Health Sci. 2010;10.

12. Leslie K. Survey sampling. New York: John Wiley and Sons, Inc. 1965:78-94.

13. Statistical package for social sciences (version 22.0).

14. Patton MQ. Qualitative evaluation and research methods. SAGE Publications Inc. 1990.

15. Onubogu CU, Ugochukwu EF, Egbuonu I, Nnamdi A, Onyeka IN, Chinyere O. Adherence to infant-feeding choices by HIVinfected mothers at a Nigerian tertiary hospital: The pre-rapid advice experience. SAJCN S Afr J Clin Nutr. 2014;28:180-186.

16. Goga AE, Doherty T, Jackson DJ, Sanders D, Colvin M, Chopra $\mathrm{M}$, et al. Infant feeding practices at routine PMTCT sites, South Africa: Results of a prospective observational study amongst HIV exposed and unexposed infants-birth to 9 months. Int Breastfeed J. 2012;7:4. 